\title{
Design, Synthesis, and In Vitro Antimicrobial Evaluation of Fused Pyrano[3,2-e]tetrazolo[1,5-c]pyrimidines and Diazepines
}

\author{
Sankari Kanakaraju, P. Sagar Vijay Kumar, Bethanamudi Prasanna, \\ and G. V. P. Chandramouli \\ Department of Chemistry, National Institute of Technology, Warangal, Andhra Pradesh 506 004, India
}

Correspondence should be addressed to G. V. P. Chandramouli; gvpc2000@gmail.com

Received 16 June 2013; Accepted 18 July 2013

Academic Editors: M. D’Auria, J. C. Menéndez, D. Sémeril, and J. Wu

Copyright (C) 2013 Sankari Kanakaraju et al. This is an open access article distributed under the Creative Commons Attribution License, which permits unrestricted use, distribution, and reproduction in any medium, provided the original work is properly cited.

A series of novel pyranochromene-containing tetrazoles fused with pyrimidinethiones, pyrimidines, and diazepines $3 \mathbf{a}-\mathbf{f}, \mathbf{4 a}-\mathbf{f}$, and $\mathbf{5 a}-\mathbf{f}$ were synthesized by condensation of the corresponding tetrazoles $\mathbf{2 a}-\mathbf{f}$ with carbon disulfide, benzaldehyde, and 4-methoxy phenacyl bromide, respectively. The compound $\mathbf{2 a}-\mathbf{f}$ was obtained by reaction of pyrano[3,2-c]chromenes $\mathbf{l a}-\mathbf{f}$ with sodium azide. The structures of the newly synthesized compounds $\mathbf{2} \mathbf{a}-\mathbf{f}$ to $\mathbf{5 a}-\mathbf{f}$ were established on the basis of their elemental analyses, IR, ${ }^{1} \mathrm{H}$ $\mathrm{NMR},{ }^{13} \mathrm{C} \mathrm{NMR}$, and mass spectral data. All of the title compounds were subjected to in vitro antibacterial testing against four pathogenic strains and antifungal screening against two fungi. Preliminary results indicate that some of them exhibited promising activities and that they deserve more consideration as potential antimicrobials.

\section{Introduction}

Pyrano[3,2-c]chromene derivatives are a class of important heterocycles with a wide range of biological properties [1] such as spasmolytic, diuretic, anticoagulant, anticancer, and antianaphylactic activities [2-5].

Tetrazoles represent an important class of heterocyclic compounds with wide ranging applications [6]. The synthesis of novel tetrazole derivatives and the investigation of their chemical and biological behavior has gained more importance in the recent decades for biological and pharmaceutical reasons. They have found use in pharmaceuticals as lipophilic spacers and carboxylic acid surrogates, which improves oral absorption [7]. Their derivatives were reported to possess broad spectrum of biological activity in both medicinal and pharmaceutical areas such as antinociceptive [8], antibacterial [9], antifungal [10], anti-HIV, anticancer, immunosuppressive [11], anti-inflammatory [12] and antiulcer [13], antiproliferative [14], antiallergic [15], and analgesic [16] activities.
On the other hand, pyrimidine scaffold was the base of many bioactive molecules such as antitubercular, antimicrobial [17], antiviral [18], antitumor [19, 20], anti-inflammatory, analgesic [21], antioxidant [22], antiproliferative [23], and antileishmanial agents [24]. Consequently, synthetic methodologies for synthesis of novel pyrimidines or pyrimidine fused compounds are of particular interests to organic and medicinal chemists.

The diazepine family represents one of the most prominent classes of privileged scaffolds in the field of drugs and pharmaceuticals. These compounds are widely used as anticonvulsant, antianxiety, analgesic, sedative, antidepressive, and hypnotic agents [25-27].

In view of the abovementioned facts, it was envisaged that these active pharmacophores, if linked together, would generate novel molecular templates which are likely to exhibit interesting biological properties. Hence, in continuation of our interest in the synthesis of biologically active heterocycles $[28,29]$, we report herein the synthesis and antimicrobial evaluation of some new heterocyclic compounds like 
pyranochromenes-containing tetrazoles. These tetrazoles are fused to six and seven-membered heterocyclic rings like pyrimidines, pyrimidinethiones, and diazepines. This combination was suggested in an attempt to investigate the influence of such structure variation on the anticipated biological activities, hoping to add some synergistic biological significance to the target molecules.

\section{Results and Discussion}

The synthetic strategies adopted for the synthesis of the target compounds are depicted in Scheme 1. The starting materials used in the present scheme, that is, pyrano[3,2-c]chromenes la-f, were prepared according to the previously reported procedure [30]. Pyrano[3,2-c]chromenes la-f were allowed to react with sodium azide in presence of $\mathrm{NH}_{4} \mathrm{Cl}$ in $\mathrm{DMF}$ to give the corresponding tetrazoles $\mathbf{2 a - f}$, which on reaction with $\mathrm{CS}_{2}$ in presence of pyridine yielded the corresponding pyrimidine-5-thiones $\mathbf{3 a}-\mathbf{f}$. Condensation of tetrazoles $\mathbf{2} \mathbf{a}-\mathbf{f}$ with benzaldehyde furnished the respective pyrimidines $\mathbf{4 a -}$ f. The compounds $\mathbf{5 a - f}$ were obtained by condensing tetrazoles 2a-f with 4-methoxyphenacyl bromide under reflux in ethanol. The structures of all of the newly synthesized compounds were elucidated on the basis of their spectral (IR, ${ }^{1} \mathrm{H}$ NMR, ${ }^{13} \mathrm{C}$ NMR, and mass) and elemental analyses data. The synthesized compounds $\mathbf{2} \mathbf{a}-\mathbf{f}, \mathbf{3} \mathbf{a}-\mathbf{f}, \mathbf{4 a}-\mathbf{f}$, and $\mathbf{5 a}-\mathbf{f}$ were also assayed for their antimicrobial activities.

The formation of tetrazoles $\mathbf{2 a - f}$ from pyrano[3,2c]chromenes la-f was confirmed by their IR, ${ }^{1} \mathrm{H}$ NMR, and ${ }^{13} \mathrm{C}$ NMR. In IR spectrum the disappearance of sharp absorption band $(-\mathrm{CN})$ around $2200 \mathrm{~cm}^{-1}$ and the appearance of band $(-\mathrm{NH})$ around $3300 \mathrm{~cm}^{-1}$ showed the formation of tetrazole, while in ${ }^{1} \mathrm{H}$ NMR the tetrazole $\mathrm{NH}$ proton was observed as a singlet at $\delta 10.01-10.21 \mathrm{ppm}$ and in ${ }^{13} \mathrm{C}$ NMR the tetrazole carbon was observed around $\delta 159.18-159.82 \mathrm{ppm}$. Furthermore, the condensation product $\mathbf{3 a - f}$ was confirmed by its IR spectrum which showed absence of the characteristic absorption band due to the $-\mathrm{NH}_{2}$ group, and according to the IR spectroscopic data of these $\mathbf{3 a - f}$ compounds which have tetrazolopyrimidin-5-thione structure, the observation of the $\mathrm{C}=\mathrm{S}$ stretching band at $1165-1181 \mathrm{~cm}^{-1}$, and the absence of an absorption band at about $2550-2600 \mathrm{~cm}^{-1}$ region cited for $\mathrm{SH}$ group have proved that these compounds were in the thionic form. In their ${ }^{13} \mathrm{C} N M R$, peak due to $\mathrm{C}=\mathrm{S}$ appeared at $\delta 189.72-190.16 \mathrm{ppm}$. The formation of $\mathbf{4 a}-\mathbf{f}$ from $\mathbf{2 a}-\mathbf{f}$ was confirmed by their IR spectra in which no $-\mathrm{NH}_{2}$ stretching vibrations were observed, and were well supported by their ${ }^{1} \mathrm{H}$ NMR and ${ }^{13} \mathrm{C}$ NMR. In ${ }^{1} \mathrm{H}$ NMR, peaks shown at $\delta$ 6.22-6.34 ppm were assigned to pyrimidine $\mathrm{CH}$ proton, and in ${ }^{13} \mathrm{C}$ NMR the signal was observed at $\delta 63.51-64.38 \mathrm{ppm}$ due to pyrimidine carbon. Other signals were found in accordance to the established structures. Furthermore, the conversion of tetrazoles $\mathbf{2 a - f}$ to fused tetrazolodiazepines 5a-f was also well confirmed by their IR, ${ }^{1} \mathrm{H}$ NMR, and ${ }^{13} \mathrm{C}$ NMR spectra. The disappearance of characteristic peaks due to $\mathrm{NH}_{2}$ and $\mathrm{NH}$ groups of $\mathbf{2 a - f}$ clearly indicated the smooth cyclization. Their ${ }^{1} \mathrm{H}$ NMR spectra showed a singlet at $\delta$ 6.13-6.32 ppm for two protons, which is attributed to the methylene protons of diazepine ring, while in ${ }^{13} \mathrm{C}$ NMR the signal that appeared at $\delta 48.43-49.06 \mathrm{ppm}$ was assigned to methylene carbon of diazepine ring. For all of the compounds, the band displayed at $1700-1719 \mathrm{~cm}^{-1}$ was due to the lactone carbonyl group, which was observed around $\delta 160.22-161.93 \mathrm{ppm}$ in ${ }^{13} \mathrm{C} \mathrm{NMR}$. The signal due to pyran proton, present in all of the compounds, appeared as a singlet at $\delta 4.56-5.12 \mathrm{ppm}$. All of the other aromatic and aliphatic protons were observed at the expected regions. Mass spectra of almost all of the compounds showed $\mathrm{M}+1$ peaks, in agreement with their molecular weights. However, in some cases, $M+2$ peaks were also observed. For all of the compounds, the elemental analyses values were in good agreement with the theoretical data. Full characterization details were provided in the Experimental section.

2.1. Antimicrobial Activity. The antibacterial activity of the synthesized compounds $\mathbf{2} \mathbf{a}-\mathbf{f}, \mathbf{3} \mathbf{a}-\mathbf{f}, \mathbf{4 a}-\mathbf{f}$, and $\mathbf{5 a - f}$ was screened against the Gram-positive bacteria such as Bacillus subtilis and Staphylococcus aureus and the Gram-negative bacteria, that is, Pseudomonas aeruginosa and Escherichia coli using nutrient agar medium. The antifungal activity of the compounds was tested against Candia albicans and Aspergillus niger using Potato dextrose agar (PDA) medium. The minimum inhibitory concentration (MIC) was carried out using microdilution susceptibility method [31]. Ciprofloxacin was used as a standard antibacterial drug, and fluconazole was used as a standard antifungal drug. The observed data on the antimicrobial activity of compounds and control drugs are given in Table 1.

The MIC values were determined as the lowest concentration that completely inhibited visible growth of the microorganisms. The investigation of antibacterial screening (Table 1) revealed that some of the newly synthesized compounds showed moderate-to-good inhibition at $25-100 \mu \mathrm{g} / \mathrm{mL}$ in DMSO. Amongst all of the compounds, compounds $\mathbf{3 b}$ and $3 \mathbf{e}$ exhibited good activities against B. subtilis (MIC: $25 \mu \mathrm{g} / \mathrm{mL}$ ) and E. coli (MIC: 50 and $25 \mu \mathrm{g} / \mathrm{mL}$ ) and moderate activities against $S$. aureus and $P$. aeruginosa. Compound $\mathbf{4 b}$ displayed good activity against $S$. aureus (MIC: $50 \mu \mathrm{g} / \mathrm{mL}$ ), whereas compound $\mathbf{4 e}$ exhibited good activity against $P$. aeuroginosa (MIC: $50 \mu \mathrm{g} / \mathrm{mL}$ ).

The investigation of antifungal screening (Table 1) revealed that some of the newly synthesized compounds showed moderate-to-good inhibition at $25-100 \mu \mathrm{g} / \mathrm{mL}$ in DMSO. Among the tested compounds, compounds $\mathbf{2 b}, \mathbf{3 b}$, and $4 \mathbf{e}$ were found to be more active than other compounds against $A$. niger (MIC: $50 \mu \mathrm{g} / \mathrm{mL}$ ). Compounds $\mathbf{2 c}, \mathbf{3 e}$, and $\mathbf{4 b}$ possess good activities against C. albicans (MIC: $50 \mu \mathrm{g} / \mathrm{mL}$ ). Compounds $\mathbf{2 f}$, 5 a, and $\mathbf{5 f}$ showed no activity against the tested species. Remaining compounds showed moderate-toleast activity against both bacteria and fungi. The structure activity relationship of the synthesized compounds revealed that the compounds having diazepine ring showed the least activity compared with other compounds. The presence of chloro and methoxy group enhances the activities of the compounds. The maximum antimicrobial activity was observed with compounds $\mathbf{3 b}, \mathbf{3 e}, \mathbf{4 b}$, and $\mathbf{4 e}$. 
<smiles>[R]c1ccc(C2C(C#N)=C(N)Oc3c2c(=O)oc2ccccc32)cc1</smiles>

1a-f<smiles>[R]Cc1ccccc1C1C(c2nnn[nH]2)=C(N)Oc2c1c(=O)oc1ccccc21</smiles>

$2 \mathbf{a}-\mathbf{f}$ (ii)<smiles>[R]Cc1ccc(C2c3c(c4ccccc4oc3=O)Oc3[nH]c(=S)n4nnnc4c32)cc1</smiles>

3a-f<smiles></smiles>

$4 a-f$<smiles>[R]Cc1ccc(C2C3=C(N=C(c4ccc(OC)cc4)Cn4nnnc43)Oc3c2c(=O)oc2ccccc32)cc1</smiles>

$5 \mathbf{a}-\mathbf{f}$

$\mathrm{R}=(\mathbf{a}) \mathrm{H},(\mathbf{b}) 4-\mathrm{Cl},(\mathbf{c}) 4-\mathrm{F}$, (d) 4-Me, (e) 4-OMe, (f) 3- $\mathrm{NO}_{2}$

Scheme 1: Schematic representation for the synthesis of fused tetrazole derivatives. Reagents and conditions: $(\mathrm{i}) \mathrm{NaN}_{3}, \mathrm{DMF}^{\mathrm{N}} \mathrm{NH}_{4} \mathrm{Cl}, 120^{\circ} \mathrm{C}$, $7 \mathrm{~h}$; (ii) $\mathrm{CS}_{2}$, pyridine, reflux, $10 \mathrm{~h}$; (iii) conc. $\mathrm{HCl}$, benzaldehyde, $\mathrm{MeOH}$, reflux, $16 \mathrm{~h}$; (iv) 4-methoxyphenacyl bromide, EtOH, AcONa, reflux, $16 \mathrm{~h}$.

\section{Conclusion}

In summary, a series of novel pyranochromene-containing fused tetrazole derivatives have been synthesized and characterized by spectral and elemental analyses. All of the newly synthesized compounds were screened for their in vitro antimicrobial activities. Among the screened samples, $\mathbf{3 b}, \mathbf{3 e}$, $\mathbf{4 b}$, and $\mathbf{4 e}$ showed significant antibacterial and antifungal activities compared with other tested samples.

\section{Experimental}

Melting points were recorded in open capillary and were uncorrected. Column chromatography was performed using silica-gel (100-200 mesh size) purchased from Thomas Baker, and thin-layer chromatography (TLC) was carried out using aluminium sheets precoated with silica-gel $60 \mathrm{~F}_{254}$ purchased from Merck. IR spectra $(\mathrm{KBr})$ were obtained using a Bruker WM-4(X) spectrometer (577 model). ${ }^{1} \mathrm{H}$ NMR $(400 \mathrm{MHz})$ and ${ }^{13} \mathrm{C}$ NMR $(100 \mathrm{MHz})$ spectra were recorded on a Bruker WM-400 spectrometer in DMSO- $d_{6}$ with TMS as an internal standard. Mass spectra (ESI) were carried out on a JEOL SX-102 spectrometer. CHN analysis was done by the Carlo Erba EA 1108 automatic elemental analyzer. The chemicals and solvents used were of commercial grade and were used without further purification unless otherwise stated.

4.1. General Procedure for the Synthesis of 2-Amino-4-aryl3-(1H-tetrazol-5-yl)pyrano[3,2-c]chromen-5(4H)-ones $(2 a-f)$. To a mixture of compound la-f $(10 \mathrm{mmol})$ in DMF $(25 \mathrm{~mL})$, sodium azide $(0.78 \mathrm{~g}, 12 \mathrm{mmol})$ and $\mathrm{NH}_{4} \mathrm{Cl}(0.64 \mathrm{~g}, 12 \mathrm{mmol})$ were added, and the reaction mixture was stirred at $120^{\circ} \mathrm{C}$ for $7 \mathrm{~h}$. The progress of the reaction was monitored by TLC. After completion of the reaction, the reaction mixture was cooled to room temperature and poured into ice cold water $(100 \mathrm{~mL})$. The solid separated was filtered, washed with water, dried, and purified by column chromatography on silica-gel using 
TABLE 1: Minimum inhibitory concentration (MIC, $\mu \mathrm{g} / \mathrm{mL}$ ) of the synthesized compounds $\mathbf{2 a}-\mathbf{f}, \mathbf{3 a}-\mathbf{f}, \mathbf{4 a}-\mathbf{f}$, and $\mathbf{5 a}-\mathbf{f}$.

\begin{tabular}{|c|c|c|c|c|c|c|}
\hline \multirow{2}{*}{ Compound } & \multicolumn{2}{|c|}{ Gram-positive bacteria } & \multicolumn{2}{|c|}{ Gram-negative bacteria } & \multicolumn{2}{|c|}{ Fungi } \\
\hline & B. subtilis & S. aureus & P. aeuroginosa & E. coli & C. albicans & A. niger \\
\hline $2 a$ & 400 & 400 & - & - & 400 & 400 \\
\hline $2 b$ & 400 & 200 & 200 & 400 & 200 & 50 \\
\hline $2 c$ & 400 & 400 & 400 & 200 & 50 & 400 \\
\hline $2 d$ & 400 & 400 & 200 & 400 & 400 & 200 \\
\hline $2 e$ & 200 & 400 & 400 & 200 & 200 & 400 \\
\hline $2 f$ & - & - & - & - & - & - \\
\hline $3 a$ & 400 & 400 & 200 & 200 & 400 & 200 \\
\hline $3 b$ & 25 & 100 & 100 & 50 & 100 & 50 \\
\hline $3 c$ & 200 & 400 & 200 & 400 & 200 & 200 \\
\hline $3 d$ & 400 & 200 & 200 & 400 & 200 & 400 \\
\hline $3 e$ & 25 & 100 & 100 & 25 & 50 & 100 \\
\hline $3 f$ & 400 & 400 & 400 & 400 & 400 & 400 \\
\hline $4 a$ & 400 & 400 & 400 & 400 & 200 & 400 \\
\hline $4 b$ & 100 & 50 & 200 & 100 & 50 & 200 \\
\hline $4 c$ & 400 & 200 & 400 & 400 & 400 & 400 \\
\hline $4 d$ & 200 & 400 & 400 & 200 & 400 & 200 \\
\hline $4 e$ & 200 & 100 & 50 & 100 & 200 & 50 \\
\hline $4 \mathrm{f}$ & 400 & 200 & 400 & 400 & 400 & 200 \\
\hline $5 a$ & - & - & - & - & - & - \\
\hline $5 b$ & 200 & 400 & - & 400 & - & 200 \\
\hline $5 c$ & - & 400 & 400 & - & 400 & 400 \\
\hline $5 d$ & 400 & 200 & 200 & - & 200 & - \\
\hline $5 e$ & 200 & 400 & 200 & 400 & 200 & 400 \\
\hline $5 f$ & - & - & - & - & - & - \\
\hline Ciprofloxacin & 6.25 & 6.25 & 6.25 & 6.25 & & \\
\hline Fluconazole & & & & & 6.25 & 6.25 \\
\hline
\end{tabular}

hexane/ethyl acetate $(7: 3)$ as eluent to afford compound $2 \mathbf{a}-$ f.

4.2. 2-Amino-4-phenyl-3-(1H-tetrazol-5-yl)pyrano[3,2-c]chromen-5(4H)-one (2a). White solid, yield $2.58 \mathrm{~g}(72 \%)$, m.p. $213-$ $216^{\circ} \mathrm{C}$; IR $\left(\mathrm{KBr}, \mathrm{cm}^{-1}\right): 3386,3278,3196,2992,1706,1660,1567$, $1497,1310,1279,1110,1051 ;{ }^{1} \mathrm{H}$ NMR $\left(400 \mathrm{MHz}, \mathrm{DMSO}-d_{6}\right): \delta$ 4.74 (s, 1H, H-4), 7.24-7.34 (m, 5H, ArH), 7.41 (brs, $2 \mathrm{H}, \mathrm{NH}_{2}$ ), 7.45-7.52 (m, 2H, ArH), 7.69-7.89 (m, 1H, ArH), 7.91 (d, 1H, ArH), $10.01(\mathrm{~s}, 1 \mathrm{H}, \mathrm{NH}) ;{ }^{13} \mathrm{C}$ NMR $\left(100 \mathrm{MHz}, \mathrm{DMSO}-d_{6}\right): \delta$ $36.96,89.03,103.97,112.93,119.21,122.43,124.62,127.09,127.61$, $128.49,132.87,143.31,152.10,153.38,157.97,159.50,161.10$; MS $m / z: 360(\mathrm{M}+1)^{+}$. Anal. Calcd for $\mathrm{C}_{19} \mathrm{H}_{13} \mathrm{~N}_{5} \mathrm{O}_{3}: \mathrm{C}, 63.51 ; \mathrm{H}$, 3.65 ; N, 19.49. Found: C, 63.62; H, 3.71; N, $19.38 \%$.

4.3. 2-Amino-4-(4-chlorophenyl)-3-(1H-tetrazol-5-yl)pyrano [3,2-c]chromen-5(4H)-one (2b). White solid, yield $2.67 \mathrm{~g}$ (68\%), m.p. $192-194^{\circ} \mathrm{C}$; IR $\left(\mathrm{KBr}, \mathrm{cm}^{-1}\right): 3397,3284,3193$, 2990, 1713, 1664, 1551, 1493, 1321, 1281, 1121, 1063; ${ }^{1} \mathrm{H}$ NMR (400 MHz, DMSO- $d_{6}$ ): $\delta 4.66$ (s, 1H, H-4), 7.30 (d, 2H, ArH), 7.36-7.44 (m, 4H, ArH + $\left.\mathrm{NH}_{2}\right), 7.48$ (d, 1H, ArH), $7.54(\mathrm{t}, 1 \mathrm{H}$, ArH), 7.78 (t, 1H, ArH), 7.92 (d, 1H, ArH), 10.14 (s, 1H, NH);
${ }^{13} \mathrm{C}$ NMR $\left(100 \mathrm{MHz}, \mathrm{DMSO}-d_{6}\right): \delta 37.12,88.12,103.89,113.88$, $119.73,123.34,125.48,129.32,130.51,132.66,133.71,143.15$, 153.14, 154.41, 158.13, 159.64, 160.25; MS m/z: $394(\mathrm{M}+1)^{+}$. Anal. Calcd for $\mathrm{C}_{19} \mathrm{H}_{12} \mathrm{ClN}_{5} \mathrm{O}_{3}: \mathrm{C}, 57.95 ; \mathrm{H}, 3.07 ; \mathrm{N}, 17.78$. Found: C, 57.83; H, 3.14; N, 17.86\%.

4.4. 2-Amino-4-(4-fluorophenyl)-3-(1H-tetrazol-5-yl)pyrano [3,2-c]chromen-5(4H)-one (2c). Brown solid, yield $2.26 \mathrm{~g}$ (60\%), m.p. $161-164^{\circ} \mathrm{C}$; IR $\left(\mathrm{KBr}, \mathrm{cm}^{-1}\right)$ : 3416, 3378, 3296, 2998, 1719, 1666, 1566, 1491, 1322, 1284, 1114, 1053; ${ }^{1} \mathrm{H}$ NMR $\left(400 \mathrm{MHz}, \mathrm{DMSO}-d_{6}\right): \delta 4.62(\mathrm{~s}, 1 \mathrm{H}, \mathrm{H}-4), 7.28(\mathrm{~d}, 2 \mathrm{H}, \mathrm{ArH})$, 7.34-7.46 (m, 4H, ArH + $\mathrm{NH}_{2}$ ), 7.50 (d, 1H, ArH), 7.58 (t, 1H, $\operatorname{ArH}), 7.75$ (t, 1H, ArH), 7.94 (d, 1H, ArH), 10.06 (s, 1H, NH); ${ }^{13} \mathrm{C}$ NMR $\left(100 \mathrm{MHz}, \mathrm{DMSO}-d_{6}\right): \delta 38.17,88.43,103.72,112.67$, $120.14,123.42,125.36,129.12,130.64,132.48,133.52,143.76$, 153.96, 154.71, 158.25, 159.73, 160.41; MS m/z: $378(\mathrm{M}+1)^{+}$. Anal. Calcd for $\mathrm{C}_{19} \mathrm{H}_{12} \mathrm{FN}_{5} \mathrm{O}_{3}$ : C, 60.48; H, 3.21; N, 18.56 . Found: C, 60.59; H, 3.26; N, 18.43\%.

4.5. 2-Amino-3-(1H-tetrazol-5-yl)-4-p-tolylpyrano[3,2-c]chromen-5(4H)-one (2d). White solid, yield 2.34 g (63\%); m.p. 208$210^{\circ} \mathrm{C}$; IR $\left(\mathrm{KBr}, \mathrm{cm}^{-1}\right)$ : 3392, 3287, 3206, 2982, 1708, 1661, 1555 , 
$1494,1319,1273,1125,1057 ;{ }^{1} \mathrm{H}$ NMR (400 MHz, DMSO- $\left.d_{6}\right)$ : $\delta 2.38\left(\mathrm{~s}, 3 \mathrm{H}, \mathrm{CH}_{3}\right), 4.63(\mathrm{~s}, 1 \mathrm{H}, \mathrm{H}-4), 7.24$ (d, 2H, ArH), 7.32-7.40 (m, 4H, ArH + $\left.\mathrm{NH}_{2}\right), 7.48(\mathrm{~d}, 1 \mathrm{H}, \mathrm{ArH}), 7.54(\mathrm{t}$, $1 \mathrm{H}, \mathrm{ArH}), 7.71$ (t, 1H, ArH), 7.84 (d, 1H, ArH), 10.02 (s, 1H, $\mathrm{NH}) ;{ }^{13} \mathrm{C}$ NMR $\left(100 \mathrm{MHz}, \mathrm{DMSO}-d_{6}\right): \delta 20.94,37.62,88.26$, $103.21,118.83,119.76,122.48,125.28,129.32,130.61,131.56$, $133.68,142.64,153.06,154.24,158.41,159.18,160.22 ; \mathrm{MS} m / z$ : $374(\mathrm{M}+1)^{+}$. Anal. Calcd for $\mathrm{C}_{20} \mathrm{H}_{15} \mathrm{~N}_{5} \mathrm{O}_{3}$ : C, 64.34; H, 4.05; N, 18.76. Found: C, 64.46; H, 4.11; N, 18.62\%.

4.6. 2-Amino-4-(4-methoxyphenyl)-3-(1H-tetrazol-5-yl)pyrano [3,2-c]chromen-5(4H)-one (2e). Orange solid, yield $2.95 \mathrm{~g}$ (76\%); m.p. 231-233 ${ }^{\circ} \mathrm{C}$; IR $\left(\mathrm{KBr}, \mathrm{cm}^{-1}\right)$ : 3419, 3376, 3284, 2992, 1716, 1668, 1566, 1492, 1320, 1283, 1117, 1061; ${ }^{1} \mathrm{H}$ NMR $\left(400 \mathrm{MHz}, \mathrm{DMSO}-d_{6}\right): \delta 3.84\left(\mathrm{~s}, 3 \mathrm{H}, \mathrm{OCH}_{3}\right), 4.71(\mathrm{~s}, 1 \mathrm{H}, \mathrm{H}-$ 4), $6.92(\mathrm{~d}, 2 \mathrm{H}, \mathrm{ArH}), 7.18-7.34\left(\mathrm{~m}, 4 \mathrm{H}, \mathrm{ArH}+\mathrm{NH}_{2}\right), 7.45$ (d, $1 \mathrm{H}, \mathrm{ArH}), 7.51$ (t, 1H, ArH), 7.70 (t, 1H, ArH), 7.89 (d, 1H, ArH), $10.16(\mathrm{~s}, 1 \mathrm{H}, \mathrm{NH}) ;{ }^{13} \mathrm{C}$ NMR $\left(100 \mathrm{MHz}, \mathrm{DMSO}-d_{6}\right): \delta 38.84$, $55.90,89.24,105.13,113.72,114.81,120.12,123.26,125.43,129.52$, $133.55,136.17,152.94,153.82,157.56,158.24,159.72,160.36$; MS $m / z: 390(\mathrm{M}+1)^{+}$. Anal. Calcd for $\mathrm{C}_{20} \mathrm{H}_{15} \mathrm{~N}_{5} \mathrm{O}_{4}: \mathrm{C}, 61.69 ; \mathrm{H}$, 3.88 ; N, 17.99. Found: C, 61.74; H, 3.77; N, 17.87\%.

4.7. 2-Amino-4-(3-nitrophenyl)-3-(1H-tetrazol-5-yl)pyrano[3, 2-c]chromen-5(4H)-one (2f). White solid, yield $2.26 \mathrm{~g}(56 \%)$; m.p. $198-200^{\circ} \mathrm{C}$; IR $\left(\mathrm{KBr}, \mathrm{cm}^{-1}\right)$ : 3408, 3388, 3294, 2990, 1718, $1663,1561,1495,1318,1264,1122,1051 ;{ }^{1} \mathrm{H}$ NMR $(400 \mathrm{MHz}$, DMSO- $\left.d_{6}\right): \delta 4.92(\mathrm{~s}, 1 \mathrm{H}, \mathrm{H}-4), 7.42-7.52(\mathrm{~m}, 4 \mathrm{H}, \mathrm{ArH}+$ $\mathrm{NH}_{2}$ ), 7.64 (t, 1H, ArH), 7.73 (t, 1H, ArH), 7.84 (d, 1H, ArH), 7.92 (d, 1H, ArH), 8.12 (d, 1H, ArH), 8.16 (s, 1H, ArH), 10.21 $(\mathrm{s}, 1 \mathrm{H}, \mathrm{NH}) ;{ }^{13} \mathrm{C}$ NMR $\left(100 \mathrm{MHz}, \mathrm{DMSO}-d_{6}\right): \delta 38.92,89.43$, 103.82 , 113.71, 119.62, 123.11, 123.23, 123.64, 125.46, 130.29, $133.69,135.36,146.61,148.27,153.13,154.56,158.81,159.82$, 160.74; MS m/z: $405(\mathrm{M}+1)^{+}$. Anal. Calcd for $\mathrm{C}_{19} \mathrm{H}_{12} \mathrm{~N}_{6} \mathrm{O}_{5}$ : C, 56.44; H, 2.99; N, 20.78. Found: C, 56.50; H, 3.08; N, $20.67 \%$.

4.8. General Procedure for the Synthesis of Fused Pyrano[3,2e]tetrazolo[1,5-c]pyrimidin-5-thiones $(3 \boldsymbol{a}-\boldsymbol{f})$. A mixture of compound $2 \mathbf{a}-\mathbf{f}(2 \mathrm{mmol})$ and carbon disulfide $(0.15 \mathrm{~g}$, $0.12 \mathrm{~mL}, 2 \mathrm{mmol})$ in pyridine $(10 \mathrm{~mL})$ was refluxed on a water bath for $10 \mathrm{~h}$. After completion of the reaction (monitored by TLC), the reaction mixture was cooled to room temperature, then poured into ice cold water $(20 \mathrm{~mL})$, and neutralized with hydrochloric acid $(1: 1)$. The solid obtained was filtered, washed with water, dried, and recrystallized from ethanol to afford compound $\mathbf{3} \mathbf{a}-\mathbf{f}$.

4.9. 14-Phenyl-5,6-dihydro-13-oxo-5H,13H,14H-[1]benzopyrano $\left[3^{\prime}, 4^{\prime}: 5,6\right]$ pyrano $[3,2-e][1,2,3,4]$ tetrazolo[1,5-c]pyrimidin-5thione (3a). White solid, yield $0.54 \mathrm{~g}(68 \%)$; m.p. $226-228^{\circ} \mathrm{C}$; IR $\left(\mathrm{KBr}, \mathrm{cm}^{-1}\right): 3399,2990,1710,1664,1598,1505,1310,1261$, 1181, 1089; ${ }^{1} \mathrm{H}$ NMR $\left(400 \mathrm{MHz}, \mathrm{DMSO}-d_{6}\right): \delta 5.03(\mathrm{~s}, 1 \mathrm{H}$, H-4), 7.09-7.57 (m, 7H, ArH), 7.87-7.95 (m, 2H, ArH), 10.09 $(\mathrm{s}, 1 \mathrm{H}, \mathrm{NH}) ;{ }^{13} \mathrm{C} \mathrm{NMR}\left(100 \mathrm{MHz}, \mathrm{DMSO}-d_{6}\right): \delta 32.45,86.53$, $104.19,118.14,123.63,124.25,126.65,127.99,128.09,132.02$, $139.88,144.59,152.11,155.59,156.08,160.12,161.93,190.02 ;$ MS $m / z: 403(\mathrm{M}+2)^{+}$. Anal. Calcd for $\mathrm{C}_{20} \mathrm{H}_{11} \mathrm{~N}_{5} \mathrm{O}_{3} \mathrm{~S}: \mathrm{C}, 59.84$; H, 2.76; N, 17.45. Found: C, 59.88; H, 2.85; N, 17.34\%.

4.10. 14-(4-Chlorophenyl)-5,6-dihydro-13-oxo-5H,13H,14H-[1] benzopyrano $\left[3^{\prime}, 4^{\prime}: 5,6\right]$ pyrano $[3,2-e][1,2,3,4]$ tetrazolo $[1,5-c]$ pyrimidin-5-thione $(\mathbf{3 b})$. White solid, yield $0.57 \mathrm{~g}(66 \%)$; m.p. $185-188^{\circ} \mathrm{C}$; IR $\left(\mathrm{KBr}, \mathrm{cm}^{-1}\right)$ : $3406,2998,1718,1668,1583$, $1508,1323,1267,1177,1082 ;{ }^{1} \mathrm{H}$ NMR (400 MHz, DMSO- $\left.d_{6}\right)$ : $\delta 4.76$ (s, 1H, H-4), 7.28 (d, 2H, ArH), 7.37 (d, 2H, ArH), $7.51(\mathrm{~d}, 1 \mathrm{H}, \mathrm{ArH}), 7.56(\mathrm{t}, 1 \mathrm{H}, \mathrm{ArH}), 7.77$ (t, 1H, ArH), 7.95 (d, 1H, ArH), 10.21 (s, 1H, NH); ${ }^{13} \mathrm{C}$ NMR $(100 \mathrm{MHz}$, DMSO- $\left.d_{6}\right): \delta 33.44,86.17,103.74,113.42,119.76,123.29,124.82$, $129.46,130.32,132.71,133.54,142.88,152.96,153.88,157.12$, 160.47, 161.12, 189.82; MS m/z: $436(\mathrm{M}+1)^{+}$. Anal. Calcd for $\mathrm{C}_{20} \mathrm{H}_{10} \mathrm{ClN}_{5} \mathrm{O}_{3} \mathrm{~S}: \mathrm{C}, 55.11 ; \mathrm{H}, 2.31 ; \mathrm{N}, 16.07$. Found: C, 55.16; $\mathrm{H}, 2.23$; N, $16.15 \%$.

4.11. 14-(4-Fluorophenyl)-5,6-dihydro-13-oxo-5H,13H,14H-[1] benzopyrano $\left[3^{\prime}, 4^{\prime}: 5,6\right]$ pyrano $[3,2-e][1,2,3,4]$ tetrazolo $[1,5-c]$ pyrimidin-5-thione (3c). White solid, yield 0.52 (63\%); m.p. $173-176^{\circ} \mathrm{C}$; IR (KBr, cm ${ }^{-1}$ ): 3386, 2985, 1712, 1666, 1577, 1512, $1321,1256,1167,1076 \mathrm{~cm}^{-1} ;{ }^{1} \mathrm{H}$ NMR (400 MHz, DMSO-d $\left.d_{6}\right)$ : $\delta 4.84$ (s, 1H, H-4), 7.36 (d, 2H, ArH), 7.48 (d, 2H, ArH), 7.54 (d, 1H, ArH), 7.62-7.74 (m, 2H, ArH), 7.94 (d, 1H, ArH), 10.34 $(\mathrm{s}, 1 \mathrm{H}, \mathrm{NH}) ;{ }^{13} \mathrm{C}$ NMR $\left(100 \mathrm{MHz}, \mathrm{DMSO}-d_{6}\right): \delta 34.21,87.34$, $103.48,112.56,120.71,123.56,124.22,128.96,130.82,132.45$, $133.68,143.18,153.27,154.40,157.57,160.19,161.56,189.93$; MS $m / z: 421(\mathrm{M}+2)^{+}$. Anal. Calcd for $\mathrm{C}_{20} \mathrm{H}_{10} \mathrm{FN}_{5} \mathrm{O}_{3} \mathrm{~S}: \mathrm{C}$, 57.28; H, 2.40; N, 16.70. Found: C, 57.39; H, 2.47; N, 16.62\%.

4.12. 14-(4-Methylphenyl)-5,6-dihydro-13-oxo-5H,13H,14H-[1] benzopyrano $\left[3^{\prime}, 4^{\prime}: 5,6\right]$-pyrano $[3,2-e][1,2,3,4]$ tetrazolo $[1,5-c]$ pyrimidin-5-thione (3d). White solid, yield $0.49 \mathrm{~g}(60 \%)$; m.p. $190-192^{\circ} \mathrm{C}$; IR $\left(\mathrm{KBr}, \mathrm{cm}^{-1}\right)$ : 3411, 2982, 1700, 1661, 1592, $1507,1313,1266,1172,1083 ;{ }^{1} \mathrm{H}$ NMR (400 MHz, DMSO- $\left.d_{6}\right)$ : $\delta 2.30\left(\mathrm{~s}, 3 \mathrm{H}, \mathrm{CH}_{3}\right), 4.91$ (s, 1H, H-4), 7.12 (d, 2H, ArH), 7.38 (d, 2H, ArH), 7.45 (d, 1H, ArH), 7.52-7.79 (m, 2H, ArH), $7.91(\mathrm{~d}, 1 \mathrm{H}, \mathrm{ArH}), 10.28$ (s, $1 \mathrm{H}, \mathrm{NH}) ;{ }^{13} \mathrm{C} \mathrm{NMR}(100 \mathrm{MHz}$, DMSO- $\left.d_{6}\right): \delta 20.96,34.52,87.64,103.18,118.36,119.82,122.57$, $125.39,125.43,130.72,131.44,133.84,142.72,153.14,154.36$, 158.74, 159.72, 161.78, 189.72; MS $m / z: 416(\mathrm{M}+1)^{+}$. Anal. Calcd for $\mathrm{C}_{21} \mathrm{H}_{13} \mathrm{~N}_{5} \mathrm{O}_{3} \mathrm{~S}$ : C, 60.71; H, 3.15; N, 16.86. Found: C, 60.84; H, 3.20; N, 16.75\%.

4.13. 14-(4-Methoxyphenyl)-5,6-dihydro-13-oxo-5H,13H,14H[1]benzopyrano $\left[3^{\prime}, 4^{\prime}: 5,6\right]$ pyrano $[3,2-e][1,2,3,4]$ tetrazolo $[1,5$ c]pyrimidin-5-thione (3e). White solid, yield $0.50 \mathrm{~g}$ (59\%); m.p. $200-203^{\circ} \mathrm{C}$; IR $\left(\mathrm{KBr}, \mathrm{cm}^{-1}\right)$ : 3393, 2994, 1711, 1653, 1588, $1503,1322,1268,1165,1081 ;{ }^{1} \mathrm{H}$ NMR $\left(400 \mathrm{MHz}, \mathrm{DMSO}-d_{6}\right)$ : $\delta 3.86\left(\mathrm{~s}, 3 \mathrm{H}, \mathrm{OCH}_{3}\right), 4.93$ (s, 1H, H-4), 6.98 (d, 2H, ArH), 7.22 (d, 2H, ArH), 7.48 (d, 1H, ArH), 7.54 (t, 1H, ArH), 7.76 (t, 1H, ArH), 7.89 (d, 1H, ArH), 10.33 (s, 1H, NH); ${ }^{13} \mathrm{C}$ NMR $\left(100 \mathrm{MHz}, \mathrm{DMSO}-d_{6}\right): \delta 34.64,55.96,88.71,105.24,113.64$, $114.78,120.22,123.64,125.49,129.31,133.57,136.23,152.88$, $153.91,158.71,159.76,159.88,161.28,189.83 ; \mathrm{MS} m / z: 432$ $(\mathrm{M}+1)^{+}$. Anal. Calcd for $\mathrm{C}_{21} \mathrm{H}_{13} \mathrm{~N}_{5} \mathrm{O}_{4} \mathrm{~S}$ : C, 58.46; H, 3.04; $\mathrm{N}, 16.23$. Found: C, 58.39; H, 3.14; N, 16.32\%. 
4.14. 14-(3-Nitrophenyl)-5,6-dihydro-13-oxo-5H,13H,14H-[1] benzopyrano $\left[3^{\prime}, 4^{\prime}: 5,6\right]$ pyrano $[3,2-e][1,2,3,4]$ tetrazolo $[1,5-c]$ pyrimidin-5-thione (3f). White solid, yield $0.45 \mathrm{~g}(51 \%)$; m.p. 211-213 ${ }^{\circ} \mathrm{C}$; IR (KBr, cm $\left.{ }^{-1}\right): 3396,2997,1716,1666,1591,1511$, $1314,1266,1180,1079 ;{ }^{1} \mathrm{H}$ NMR $\left(400 \mathrm{MHz}, \mathrm{DMSO}-d_{6}\right): \delta 5.10$ (s, 1H, H-4), 7.44 (d, 1H, ArH), 7.51 (t, 1H, ArH), 7.62 (t, 1H, ArH), 7.75 (t, 1H, ArH), 7.82 (d, 1H, ArH), 7.94 (d, 1H, ArH), 8.10 (d, 1H, ArH), 8.18 (s, 1H, ArH), 10.48 (s, 1H, NH); ${ }^{13} \mathrm{C}$ NMR $\left(100 \mathrm{MHz}, \mathrm{DMSO}-d_{6}\right): \delta 34.79,88.76,103.93,113.62$, $119.84,123.32,123.43,123.79,125.62,130.71,133.82,135.43$, $146.67,148.54,153.12,155.93,159.72,160.74,161.87,190.16$; MS $m / z: 447(\mathrm{M}+1)^{+}$. Anal. Calcd for $\mathrm{C}_{20} \mathrm{H}_{10} \mathrm{~N}_{6} \mathrm{O}_{5} \mathrm{~S}: \mathrm{C}, 53.81$; H, 2.26; N, 18.83. Found: C, 53.92; H, 2.35; N, 18.78\%.

4.15. General Procedure for the Synthesis of Fused Pyrano[3,2e]tetrazolo[1,5-c]pyrimidines $(\mathbf{4 a}-\boldsymbol{f})$. To a mixture of compound $2 \mathbf{a}-\mathbf{f}(2 \mathrm{mmol})$ and benzaldehyde $(0.212 \mathrm{~g}, 0.20 \mathrm{~mL}$, $2 \mathrm{mmol})$ in methanol $(10 \mathrm{~mL})$, conc. $\mathrm{HCl}(0.5 \mathrm{~mL})$ was added, and the reaction mixture was refluxed for $16 \mathrm{~h}$. The progress of the reaction was monitored by TLC. After completion of the reaction, the reaction mixture was cooled to room temperature and neutralized with saturated sodium bicarbonate solution; the solid separated was filtered, washed with water, dried, and recrystallized from ethanol to afford compound 4a-f.

4.16. 5,14-Diphenyl-6-hydro-13-oxo-5H,13H,14H-[1]benzopyrano $\left[3^{\prime}, 4^{\prime}: 5,6\right]$ pyrano $[3,2-e][1,2,3,4]$ tetrazolo $[1,5-c]$ pyrimidine (4a). White solid, yield $0.54 \mathrm{~g}(61 \%)$; m.p. $168-170^{\circ} \mathrm{C}$; IR $\left(\mathrm{KBr}, \mathrm{cm}^{-1}\right): 3384,2997,1714,1635,1593,1368,1261,1123$; ${ }^{1} \mathrm{H}$ NMR (400 MHz, DMSO- $d_{6}$ ): $\delta 4.58$ (s, $\left.1 \mathrm{H}, \mathrm{H}-4\right), 6.32(\mathrm{~s}$, $1 \mathrm{H}, \mathrm{CH}), 7.14-7.58(\mathrm{~m}, 11 \mathrm{H}, \mathrm{ArH}), 7.82-7.86(\mathrm{~m}, 2 \mathrm{H}, \mathrm{ArH})$, $7.94(\mathrm{~d}, 1 \mathrm{H}, \mathrm{ArH}), 9.60(\mathrm{~s}, 1 \mathrm{H}, \mathrm{NH}) ;{ }^{13} \mathrm{C} \mathrm{NMR}(100 \mathrm{MHz}$, DMSO- $\left.d_{6}\right): \delta 35.96,63.51,88.32,103.86,113.74,118.44,123.30$, $123.41,123.90,124.00,125.27,126.63,127.87,131.53,131.85$, 140.57, 143.77, 152.24, 153.69, 156.82, 159.68, 160.36; MS $m / z$ : $448(\mathrm{M}+1)^{+}$. Anal. Calcd for $\mathrm{C}_{26} \mathrm{H}_{17} \mathrm{~N}_{5} \mathrm{O}_{3}$ : C, 69.79; $\mathrm{H}, 3.83$; N, 15.65. Found: C, 69.72; H, 3.92; N, 15.57\%.

4.17. 14-(4-Chlorophenyl)-5-phenyl-6-hydro-13-oxo-5H,13H,14H[1] benzopyrano $\left[3^{\prime}, 4^{\prime}: 5,6\right]$ pyrano $[3,2-e][1,2,3,4]$ tetrazolo $[1,5$ c]pyrimidine (4b). White solid, yield $0.51 \mathrm{~g}$ (53\%); m.p. $155-158^{\circ} \mathrm{C}$; IR $\left(\mathrm{KBr}, \mathrm{cm}^{-1}\right)$ : 3391, 2982, 1717, 1665, 1571, 1372, 1258, 1132; ${ }^{1} \mathrm{H}$ NMR (400 MHz, DMSO- $\left.d_{6}\right): \delta 4.78(\mathrm{~s}, 1 \mathrm{H}$, H-4), 6.24 (s, 1H, CH), 7.10-7.46 (m, 9H, ArH), 7.52 (d, 1H, ArH), 7.58 (t, 1H, ArH), 7.79 (t, 1H, ArH), 7.94 (d, 1H, ArH), $9.93(\mathrm{~s}, 1 \mathrm{H}, \mathrm{NH}) ;{ }^{13} \mathrm{C}$ NMR $\left(100 \mathrm{MHz}, \mathrm{DMSO}-d_{6}\right): \delta 36.24$, $63.72,88.41,104.76,114.54,118.73,124.44,125.59,126.68$, $127.82,129.46,130.28,131.47,132.61,133.48,138.12,142.23$, 153.17, 154.53, 156.22, 159.83, 160.48; MS m/z: $482(\mathrm{M}+1)^{+}$. Anal. Calcd for $\mathrm{C}_{26} \mathrm{H}_{16} \mathrm{ClN}_{5} \mathrm{O}_{3}$ : C, 64.80; H, 3.35; N, 14.53. Found: C, 64.86; H, 3.46; N, 14.44\%.

4.18. 14-(4-Fluorophenyl)-5-phenyl-6-hydro-13-oxo-5H,13H,14H[1] benzopyrano $\left[3^{\prime}, 4^{\prime}: 5,6\right]$ pyrano $[3,2-e][1,2,3,4]$ tetrazolo $[1,5$ c]pyrimidine $(\mathbf{4 c})$. Brown solid, yield $0.49 \mathrm{~g}$ (53\%); m.p. 187-189 ${ }^{\circ}$; IR (KBr, cm ${ }^{-1}$ ): 3387, 2987, 1711, 1658, 1589, 1366, 1267, $1129 ;{ }^{1} \mathrm{H}$ NMR $\left(400 \mathrm{MHz}, \mathrm{DMSO}-d_{6}\right): \delta 4.83(\mathrm{~s}, 1 \mathrm{H}$,
$\mathrm{H}-4), 6.22$ (s, 1H, CH), 7.12-7.45 (m, 9H, ArH), 7.56 (d, 1H, ArH), 7.63 (t, 1H, ArH), 7.74 (t, 1H, ArH), 7.96 (d, 1H, ArH), $10.10(\mathrm{~s}, 1 \mathrm{H}, \mathrm{NH}) ;{ }^{13} \mathrm{C}$ NMR $\left(100 \mathrm{MHz}, \mathrm{DMSO}-d_{6}\right): \delta 36.48$, $63.76,88.53,103.74,113.78,119.94,122.53,124.64,126.72$, $127.39,129.65,130.48,131.62,132.73,133.66,139.12,143.13$, 153.70, 154.49, 157.11, 159.32, 160.53; MS m/z: $466(\mathrm{M}+1)^{+}$. Anal. Calcd for $\mathrm{C}_{26} \mathrm{H}_{16} \mathrm{FN}_{5} \mathrm{O}_{3}$ : C, 67.09; H, 3.46; N, 15.05. Found: C, 67.18; H, 3.37; N, 15.10\%.

4.19. 14-(4-Methylphenyl)-5-phenyl-6-hydro-13-oxo-5H,13H,14H[1]benzopyrano $\left[3^{\prime}, 4^{\prime}: 5,6\right]$ pyrano $[3,2-e][1,2,3,4]$ tetrazolo $[1,5$ c]pyrimidine $(\mathbf{4 d})$. White solid, yield $0.53 \mathrm{~g}$ (58\%); m.p. $174-176^{\circ} \mathrm{C}$; IR $\left(\mathrm{KBr}, \mathrm{cm}^{-1}\right): 3371,2982,1706,1669,1584,1371$, 1256,$1125 ;{ }^{1} \mathrm{H}$ NMR $\left(400 \mathrm{MHz}, \mathrm{DMSO}-d_{6}\right): \delta 2.32(\mathrm{~s}, 3 \mathrm{H}$, $\left.\mathrm{CH}_{3}\right), 4.96$ (s, 1H, H-4), $6.28(\mathrm{~s}, 1 \mathrm{H}, \mathrm{CH}), 7.08-7.40(\mathrm{~m}, 9 \mathrm{H}$, ArH), 7.46 (d, 1H, ArH), 7.56 (t, 1H, ArH), 7.71 (t, 1H, ArH), 7.93 (d, 1H, ArH), 10.18 (s, 1H, NH); ${ }^{13} \mathrm{C}$ NMR $(100 \mathrm{MHz}$, DMSO- $\left.d_{6}\right): \delta 20.96,37.08,64.16,88.72,103.32,118.78,119.72$, $122.53,125.36,126.57,128.71,129.43,130.53,132.19,133.58$, 138.23, 139.21, 143.13, 153.70, 154.49, 157.11, 159.32, 160.57; MS $m / z: 462(\mathrm{M}+1)^{+}$. Anal. Calcd for $\mathrm{C}_{27} \mathrm{H}_{19} \mathrm{~N}_{5} \mathrm{O}_{3}: \mathrm{C}, 70.27$; $\mathrm{H}, 4.15$; N, 15.18. Found: C, 70.33; H, 4.24; N, 15.10\%.

4.20. 14-(4-Methoxyphenyl)-5-phenyl-6-hydro-13-oxo-5H,13H, $14 \mathrm{H}$-[1]benzopyrano $\left[3^{\prime}, 4^{\prime}: 5,6\right]$ pyrano[3,2-e][1,2,3,4]tetrazolo [1,5-c]pyrimidine (4e). White solid, yield $0.57 \mathrm{~g}(60 \%)$; m.p. $165-168^{\circ} \mathrm{C}$; IR $\left(\mathrm{KBr}, \mathrm{cm}^{-1}\right)$ : 3388, 2997, 1701, 1665, 1591, 1360, 1267,$1129 ;{ }^{1} \mathrm{H}$ NMR $\left(400 \mathrm{MHz}, \mathrm{DMSO}-d_{6}\right): \delta 3.85(\mathrm{~s}, 3 \mathrm{H}$, $\left.\mathrm{OCH}_{3}\right), 4.98(\mathrm{~s}, 1 \mathrm{H}, \mathrm{H}-4), 6.30(\mathrm{~s}, 1 \mathrm{H}, \mathrm{CH}), 7.01-7.44(\mathrm{~m}, 9 \mathrm{H}$, ArH), 7.50 (d, 1H, ArH), 7.54 (t, 1H, ArH), 7.73 (t, 1H, ArH), 7.84 (d, 1H, ArH), 10.14 (s, 1H, NH); ${ }^{13} \mathrm{C}$ NMR $(100 \mathrm{MHz}$, DMSO- $\left.d_{6}\right): \delta 37.43,55.48,64.34,88.76,104.07,112.39,114.73$, 120.32 , 123.59, 125.14, 126.37, 127.68, 129.38, 131.73, 133.81, $137.70,138.32,152.72,154.68,156.36,158.42,159.58,160.53$; MS $m / z: 478(\mathrm{M}+1)^{+}$. Anal. Calcd for $\mathrm{C}_{27} \mathrm{H}_{19} \mathrm{~N}_{5} \mathrm{O}_{4}: \mathrm{C}, 67.92 ; \mathrm{H}$, 4.01; N, 14.67. Found: C, 67.81; H, 4.06; N, 14.58\%.

4.21. 14-(3-Nitrophenyl)-5-phenyl-6-hydro-13-oxo-5H,13H,14H[1]benzopyrano $\left[3^{\prime}, 4^{\prime}: 5,6\right]$ pyrano $[3,2-e][1,2,3,4]$ tetrazolo $[1,5$ c]pyrimidine (4f). Yellow solid, yield $0.50 \mathrm{~g}$ (51\%); m.p. 191-194 ${ }^{\circ}$ C; IR $\left(\mathrm{KBr}, \mathrm{cm}^{-1}\right)$ : 3399, 2982, 1718, 1659, 1582, 1368, 1251,$112 ;{ }^{1} \mathrm{H}$ NMR $\left(400 \mathrm{MHz}, \mathrm{DMSO}-d_{6}\right): \delta 5.12(\mathrm{~s}, 1 \mathrm{H}, \mathrm{H}-4)$, 6.34 (s, 1H, CH), 7.11-7.38 (m, 5H, ArH), 7.46 (d, 1H, ArH), 7.53 (t, 1H, ArH), 7.60 (t, 1H, ArH), 7.76 (t, 1H, ArH), 7.88 (d, $1 \mathrm{H}, \mathrm{ArH}), 7.94$ (d, 1H, ArH), 8.12 (d, 1H, ArH), 8.20 (s, 1H, ArH) $10.24(\mathrm{~s}, 1 \mathrm{H}, \mathrm{NH}) ;{ }^{13} \mathrm{C}$ NMR $\left(100 \mathrm{MHz}, \mathrm{DMSO}-d_{6}\right): \delta$ $37.82,64.38,88.83,104.29,113.68,120.07,123.33,124.12,125.57$, $126.37,127.41,128.03,131.32,132.12,133.73,136.51,138.56$, $145.87,148.42,153.32,154.66,158.27,159.87,160.67$; MS $m / z$ : $493(\mathrm{M}+1)^{+}$. Anal. Calcd for $\mathrm{C}_{26} \mathrm{H}_{16} \mathrm{~N}_{6} \mathrm{O}_{5}$ : C, 63.41; H, 3.27; $\mathrm{N}, 17.07$. Found: C, 63.48; $\mathrm{H}, 3.16 ; \mathrm{N}, 17.16 \%$.

4.22. General Procedure for the Synthesis of Fused Pyrano[3,2e]tetrazolo[1,5-c][1,4]diazepines $(5 \boldsymbol{a}-\boldsymbol{f})$. A mixture of compound 2a-f ( $2 \mathrm{mmol}), 4$-methoxyphenacyl bromide $(0.45 \mathrm{~g}$, $2 \mathrm{mmol})$, and sodium acetate $(0.19 \mathrm{~g}, 2.4 \mathrm{mmol})$ in ethanol $(15 \mathrm{~mL})$ was refluxed for $16 \mathrm{~h}$. After completion of the reaction (monitored by TLC), the reaction mixture was cooled to 
room temperature and then poured into water $(30 \mathrm{~mL})$. The solid separated was filtered, washed with water, dried, and purified by column chromatography on silica-gel using hexane/ethyl acetate $(6: 4)$ as eluent to obtain compound $5 \mathbf{a}-$ f.

4.23. 15-Phenyl-6-(4-methoxyphenyl)-13-oxo-14H,15H-[1]benzopyrano $\left[3^{\prime}, 4^{\prime}: 5,6\right]$ pyrano[3,2-e] $[1,2,3,4]$ tetrazolo $[1,5-c][1,4]$ diazepine (5a). White solid, yield 0.56 g (58\%); m.p. 138$140^{\circ} \mathrm{C}$; IR $\left(\mathrm{KBr}, \mathrm{cm}^{-1}\right): 2954,1700,1634,1608,1509,1364$, $1288,1138,1108 ;{ }^{1} \mathrm{H}$ NMR $\left(400 \mathrm{MHz}, \mathrm{DMSO}-d_{6}\right): \delta 3.85(\mathrm{~s}$, $3 \mathrm{H}, \mathrm{OCH}_{3}$ ), 4.58 (s, 1H, H-4), 6.13 (s, 2H, CH $\left.\mathrm{CH}_{2}\right), 7.10-7.15$ (m, 2H, ArH), 7.28 (d, 2H, ArH), 7.37 (d, 2H, ArH), 7.43-7.53 (m, 3H, ArH), 7.70-7.77 (m, 2H, ArH), 7.82 (d, 1H, ArH), 7.93 (d, $1 \mathrm{H}, \mathrm{ArH}) ;{ }^{13} \mathrm{C}$ NMR $\left(100 \mathrm{MHz}, \mathrm{DMSO}-d_{6}\right): \delta 37.02$, 48.43, 55.13, 103.90, 105.73, 113.01, 118.67, 119.21, 122.48, 124.61, $126.02,126.25,127.08,128.22,129.23,132.85,142.14,152.14$, $153.35,157.22,157.80,159.53,160.22,161.87$; MS m/z: 490 $(\mathrm{M}+1)^{+}$. Anal. Calcd for $\mathrm{C}_{28} \mathrm{H}_{19} \mathrm{~N}_{5} \mathrm{O}_{4}: \mathrm{C}, 68.71 ; \mathrm{H}, 3.91 ; \mathrm{N}$, 14.31. Found: C, $68.80 ; \mathrm{H}, 3.83$; N, $14.36 \%$.

4.24. 15-(4-Chlorophenyll-6-(4-methoxyphenyl)-13-oxo-14H,15H[1] benzopyrano $\left[3^{\prime}, 4^{\prime}: 5,6\right]$ pyrano $[3,2-e][1,2,3,4]$ tetrazolo $[1,5-$ c] $[1,4]$ diazepine $(5 \boldsymbol{b})$. White solid, yield $0.54 \mathrm{~g}$ (52\%); m.p. $154-156^{\circ} \mathrm{C}$; IR $\left(\mathrm{KBr}, \mathrm{cm}^{-1}\right): 2973,1707,1651,1591,1511,1371$, $1272,1129,1112 ;{ }^{1} \mathrm{H}$ NMR $\left(400 \mathrm{MHz}, \mathrm{DMSO}-d_{6}\right): \delta 3.86(\mathrm{~s}$, $\left.3 \mathrm{H}, \mathrm{OCH}_{3}\right), 4.56$ (s, 1H, H-4), 6.17 (s, 2H, $\left.\mathrm{CH}_{2}\right), 6.98$ (d, 2H, ArH), 7.29 (d, 2H, ArH), 7.44 (d, 1H, ArH), 7.56 (d, 2H, $\operatorname{ArH}), 7.61$ (t, 1H, ArH), 7.72 (t, 1H, ArH), 7.84 (d, 2H, ArH) $7.98(\mathrm{~d}, 1 \mathrm{H}, \mathrm{ArH}) ;{ }^{13} \mathrm{C}$ NMR $\left(100 \mathrm{MHz}, \mathrm{DMSO}-d_{6}\right): \delta 37.12$, 48.51, 55.76, 104.23, 105.84, 113.14, 114.32, 119.71, 123.81, 124.93, $126.42,128.72,130.02,130.86,132.54,133.67,142.24,152.28$, $153.76,156.92,157.38,159.63,160.32,161.78 ; \mathrm{MS} m / z: 524$ $(\mathrm{M}+1)^{+}$. Anal. Calcd for $\mathrm{C}_{28} \mathrm{H}_{18} \mathrm{ClN}_{5} \mathrm{O}_{4}$ : C, 64.19; H, 3.46; $\mathrm{N}, 13.37$. Found: C, 64.24; H, 3.57; N, 13.31\%.

4.25. 15-(4-Fluorophenyl)-6-(4-methoxyphenyl)-13-oxo-14H,15H[1] benzopyrano $\left[3^{\prime}, 4^{\prime}: 5,6\right]$ pyrano[3,2-e] $[1,2,3,4]$ tetrazolo $[1,5$ c][1,4]diazepine (5c). White solid, yield $0.51 \mathrm{~g}$ (51\%); m.p. $173-176^{\circ} \mathrm{C}$; IR $\left(\mathrm{KBr}, \mathrm{cm}^{-1}\right): 2989,1704,1648,1606,1512,1371$, 1266, 1134, 1104; ${ }^{1} \mathrm{H}$ NMR $\left(400 \mathrm{MHz}, \mathrm{DMSO}-d_{6}\right): \delta 3.86$ $\left(\mathrm{s}, 3 \mathrm{H}, \mathrm{OCH}_{3}\right), 4.64$ (s, 1H, H-4), $6.19\left(\mathrm{~s}, 2 \mathrm{H}, \mathrm{CH}_{2}\right), 6.96$ (d, 2H, ArH), 7.32 (d, 2H, ArH), 7.49 (d, 1H, ArH), 7.54 (d, 2H, ArH), 7.65 (t, 1H, ArH), 7.71 (t, 1H, ArH), 7.87 (d, 2H, ArH) $7.94(\mathrm{~d}, 1 \mathrm{H}, \mathrm{ArH}) ;{ }^{13} \mathrm{C}$ NMR $\left(100 \mathrm{MHz}, \mathrm{DMSO}-d_{6}\right): \delta$ 37.43, 48.62, 55.81, 103.92, 105.74, 112.86, 114.36, 119.93, 123.25, $125.61,126.32,129.23,130.13,130.87,132.85,133.26,143.17$, $152.28,153.70,156.87,157.47,159.44,160.53,161.49 ; \mathrm{MS} m / z$ : $508(\mathrm{M}+1)^{+}$. Anal. Calcd for $\mathrm{C}_{28} \mathrm{H}_{18} \mathrm{FN}_{5} \mathrm{O}_{4}: \mathrm{C}, 66.27 ; \mathrm{H}$, 3.58 ; N, 13.80. Found: C, 66.21; H, 3.67; N, 13.88\%.

4.26. 15-(4-Methylphenyl)-6-(4-Methoxyphenyl)-13-oxo-14H,15H[1]benzopyrano $\left[3^{\prime}, 4^{\prime}: 5,6\right]$ pyrano $[3,2-e][1,2,3,4]$ tetrazolo $[1,5$ c] [1,4]diazepine (5d). White solid, yield $0.55 \mathrm{~g}$ (55\%); m.p. $165-168^{\circ} \mathrm{C}$; IR $\left(\mathrm{KBr}, \mathrm{cm}^{-1}\right)$ : 2983, 1709, 1641, 1614, 1511, 1360, 1273, 1131, 1109; ${ }^{1} \mathrm{H}$ NMR (400 MHz, DMSO- $\left.d_{6}\right): \delta 2.32(\mathrm{~s}$, $\left.3 \mathrm{H}, \mathrm{CH}_{3}\right), 3.89\left(\mathrm{~s}, 3 \mathrm{H}, \mathrm{OCH}_{3}\right), 4.68$ (s, $\left.1 \mathrm{H}, \mathrm{H}-4\right), 6.22(\mathrm{~s}, 2 \mathrm{H}$, $\mathrm{CH}_{2}$ ), 6.98 (d, 2H, ArH), 7.29-7.35 (m, 4H, ArH), 7.48 (d,
1H, ArH), 7.56 (t, 1H, ArH), 7.72 (t, 1H, ArH), 7.89 (d, 2H, ArH) 7.98 (d, 1H, ArH); ${ }^{13} \mathrm{C}$ NMR $\left(100 \mathrm{MHz}, \mathrm{DMSO}-d_{6}\right): \delta$ 20.94, 38.23, 48.71, 55.90, 103.53, 105.76, 114.47, 118.39, 119.68, $122.85,125.83,126.41,129.26,130.31,130.72,131.66,133.53$, $142.87,153.12,154.43,156.93,157.59,159.52,160.12,161.23$; MS $m / z: 504(\mathrm{M}+1)^{+}$. Anal. Calcd for $\mathrm{C}_{29} \mathrm{H}_{21} \mathrm{~N}_{5} \mathrm{O}_{4}: \mathrm{C}, 69.18 ; \mathrm{H}$, 4.20 ; N, 13.91. Found: C, 69.27; H, 4.25; N, 13.81\%.

4.27. 6,15-Di-(4-methoxyphenyl)-13-oxo-14H,15H-[1]benzopyrano $\left[3^{\prime}, 4^{\prime}: 5,6\right]$ pyrano[3,2-e][1,2,3,4] tetrazolo[1,5-c][1,4]diazepine (5e). White solid, yield $0.59 \mathrm{~g}$ (57\%); m.p. 201-203 ${ }^{\circ} \mathrm{C}$; IR $\left(\mathrm{KBr}, \mathrm{cm}^{-1}\right)$ : 2991, 1711, 1667, 1600, 1521, 1371, 1265, 1142, 1101; ${ }^{1} \mathrm{H}$ NMR (400 MHz, DMSO- $\left.d_{6}\right): \delta 3.84\left(\mathrm{~s}, 3 \mathrm{H}, \mathrm{OCH}_{3}\right), 3.89$ (s, 3H, $\left.\mathrm{OCH}_{3}\right), 4.77$ (s, $\left.1 \mathrm{H}, \mathrm{H}-4\right), 6.28\left(\mathrm{~s}, 2 \mathrm{H}, \mathrm{CH}_{2}\right), 6.98-7.10$ (m, 4H, ArH), 7.39 (d, 2H, ArH), 7.48 (d, 1H, ArH), 7.54 (t, $1 \mathrm{H}, \mathrm{ArH}), 7.70$ (t, 1H, ArH), 7.81 (d, 2H, ArH) 7.93 (d, 1H, $\mathrm{ArH}) ;{ }^{13} \mathrm{C}$ NMR $\left(100 \mathrm{MHz}, \mathrm{DMSO}-d_{6}\right): \delta 38.41,48.83,55.89$, $55.96,105.21,105.93,113.78,114.82,120.23,123.71,125.38$, $127.08,129.37,130.43,133.62,136.81,152.73,153.88,155.13$, $156.22,157.83,158.34,159.77,160.43,161.76 ; \mathrm{MS} \mathrm{m} / z 520$ $(\mathrm{M}+1)^{+}$. Anal. Calcd for $\mathrm{C}_{29} \mathrm{H}_{21} \mathrm{~N}_{5} \mathrm{O}_{5}: \mathrm{C}, 67.05 ; \mathrm{H}, 4.07 ; \mathrm{N}$, 13.48. Found: C, 67.11; H, 4.14; N, 13.39\%.

4.28. 15-(3-Nitrophenyl)-6-(4-methoxyphenyl)-13-oxo-14H,15H[1]benzopyrano $\left[3^{\prime}, 4^{\prime}: 5,6\right]$ pyrano $[3,2-e][1,2,3,4]$ tetrazolo $[1,5$ c][1,4]diazepine (5f). White solid, yield $0.51 \mathrm{~g}$ (48\%); m.p. 228-230 ${ }^{\circ}$; IR $\left(\mathrm{KBr}, \mathrm{cm}^{-1}\right): 2987,1708,1658,1603,1501,1376$, 1263, 1127, 1118; ${ }^{1} \mathrm{H}$ NMR (400 MHz, DMSO-d 6 ): $\delta 3.89(\mathrm{~s}$, $3 \mathrm{H}, \mathrm{OCH}_{3}$ ), 4.93 (s, 1H, H-4), 6.32 (s, 2H, $\mathrm{CH}_{2}$ ), 7.00 (d, $2 \mathrm{H}$, ArH), 7.41 (d, 1H, ArH), 7.51 (t, 1H, ArH), 7.63 (t, 1H, ArH), 7.75 (t, 1H, ArH), 7.80 (d, 1H, ArH), 7.89 (d, 2H, ArH), 7.98 (d, 1H, ArH), 8.10 (d, 1H, ArH), 8.16 (s, 1H, ArH); ${ }^{13} \mathrm{C}$ NMR $\left(100 \mathrm{MHz}, \mathrm{DMSO}-d_{6}\right): \delta 38.71,49.06,55.98,104.74,105.91$, $113.82,114.67,119.58,123.62,123.94,124.21,125.64,126.91$, $130.39,131.13,133.64,135.67,146.72,148.31,153.32,154.66$, 157.38, 158.32, 159.84, 160.77, 161.83; MS m/z: $535(\mathrm{M}+1)^{+}$. Anal. Calcd for $\mathrm{C}_{28} \mathrm{H}_{18} \mathrm{~N}_{6} \mathrm{O}_{6}: \mathrm{C}, 62.92 ; \mathrm{H}, 3.39 ; \mathrm{N}, 15.72$. Found: C, 62.81; H, 3.45; N, 15.62\%.

\section{Biological Protocol}

5.1. Antimicrobial Activity. All of the newly synthesized compounds $\mathbf{2} \mathbf{a}-\mathbf{f}, \mathbf{3} \mathbf{a}-\mathbf{f}, \mathbf{4 a}-\mathbf{f}$, and $\mathbf{5} \mathbf{a}-\mathbf{f}$ were screened for their antibacterial activities against the Gram-positive bacteria (Bacillus subtilis and Staphylococcus aureus) and the Gramnegative bacteria (Pseudomonas aeuroginosa and Escherichia coli). The antifungal activity of the compounds was assayed against Candia albicans and Aspergillus niger. The MICs of the compound assays were carried out using the microdilution susceptibility method. Ciprofloxacin was used as a reference antibacterial agent. Fluconazole was used as a reference antifungal agent. The test compounds, ciprofloxacin and fluconazole, were dissolved in DMSO at concentration of $800 \mu \mathrm{g} / \mathrm{mL}$, and they were then diluted in culture medium (nutrient agar for bacteria and Potato dextrose agar for fungi), and two-fold serial dilution of the solution was prepared (400, $200,100,50,12.5$, and $6.25 \mu \mathrm{g} / \mathrm{mL})$. The tubes were incubated at $36^{\circ} \mathrm{C}$ for $24 \mathrm{~h}$ and $48 \mathrm{~h}$ for bacteria and fungi, respectively. 
The minimum inhibitory concentrations (MICs, $\mu \mathrm{g} / \mathrm{mL}$ ) of the compounds were recorded as the lowest concentration of each chemical compound in the tubes with no turbidity (i.e., no growth) of inoculated bacteria/fungi.

\section{Acknowledgment}

The authors are thankful to the Director of the National Institute of Technology, Warangal, India, for providing research facilities and financial support.

\section{References}

[1] G. R. Green, J. M. Evans, A. K. Vong, A. R. Katritzky, C. W. Rees, and E. F. Scriven, Comprehensive Heterocyclic Chemistry, vol. 5, Pergamon Press, Oxford, UK, 1995.

[2] W. O. Foye, Principi Di Chemico Frmaceutica, Piccin, Padova, Italy, 1991.

[3] L. Bonsignore, G. Loy, D. Secci, and A. Calignano, "Synthesis and pharmacological activity of 2-oxo-( $2 H$ ) 1-benzopyran-3carboxamide derivatives," European Journal of Medicinal Chemistry, vol. 28, no. 6, pp. 517-520, 1993.

[4] L. L. Andreani and E. Lapi, "On some new esters of coumarin3-carboxylic acid wit balsamic and bronchodilator action," Bollettino Chimico Farmaceutico, vol. 99, pp. 583-586, 1960.

[5] E. C. Witte, P. Neubert, and A. Roesch, "7-(piperazinyl propoxy)-2H-1-benzopyran-2-ones," Ger. Offen DE, 3427985, Chemical Abstracts 104, 224915f, 1986.

[6] R. N. Butler, A. R. Katritzky, C. W. Rees, and E. F. V. Scriven, Comprehensive Heterocyclic Chemistry, vol. 4, Pergamon Press, Oxford, UK, 1996.

[7] R. J. Herr, "5-Substituted-1H-tetrazoles as carboxylic acid isosteres: medicinal chemistry and synthetic methods," Bioorganic and Medicinal Chemistry, vol. 10, no. 11, pp. 3379-3393, 2002.

[8] A. Rajasekaran and P. P. Thampi, "Synthesis and antinociceptive activity of some substituted-5-[2-(1,2,3,4- tetrahydrocarbazol9-yl)ethyl]tetrazol-1-ylalkanones," European Journal of Medicinal Chemistry, vol. 40, no. 12, pp. 1359-1364, 2005.

[9] R. M. Demarinis, J. R. E. Hoover, and G. L. Dunn, "A new parenteral cephalosporin. SK\&F 59962: 7 trifluoromethylthioacetamido 3 ( 1 methyl $1 H$ tetrazol 5 ylthiomethyl) 3 cephem 4 carboxylic acid. Chemistry and structure activity relationships," Journal of Antibiotics, vol. 28, no. 6, pp. 463-470, 1975.

[10] R. S. Upadhayaya, S. Jain, N. Sinha, N. Kishore, R. Chandra, and S. K. Arora, "Synthesis of novel substituted tetrazoles having antifungal activity," European Journal of Medicinal Chemistry, vol. 39, no. 7, pp. 579-592, 2004.

[11] A. Dlugosz, "Synthesis and biological activity of pyrimidobenzodiazepine derivatives. New ring systems: triazolo- and tetrazolo-pyrimidobenzodiazepines," Pharmazie, vol. 50, no. 3 , pp. 180-182, 1995.

[12] E. E. Knaus and P. Kumar, "Synthesis and antiinflammatory activity of 5-(1,2-dihydropyridyl)-tetrazol-2-acetic acids, esters and amides," European Journal of Medicinal Chemistry, vol. 28, no. 11, pp. 881-885, 1993.

[13] K. Terashima, T. Tanimura, H. Shimamura et al., "Studies on antiulcer agents. II. Antiulcer properties of N-(1H-tetrazol-5yl)-2-anilino-5-pyrimidinecarboxamides inhibiting release of histamine from passively sensitized rat peritoneal mast cells,"
Chemical and Pharmaceutical Bulletin, vol. 43, no. 6, pp. 10421044, 1995.

[14] A. S. Gundugola, K. L. Chandra, E. M. Perchellet, A. M. Waters, J.-P. H. Perchellet, and S. Rayat, "Synthesis and antiproliferative evaluation of 5-oxo and 5-thio derivatives of 1,4-diaryl tetrazoles," Bioorganic and Medicinal Chemistry Letters, vol. 20, no. 13, pp. 3920-3924, 2010.

[15] A. Nohara, H. Kuriki, T. Saijo, H. Sugihara, M. Kanno, and Y. Sanno, "Studies on antianaphylactic agents. 5. Synthesis of 3-(1H-tetrazol-5-yl)chromones, a new series of antiallergic substances," Journal of Medicinal Chemistry, vol. 20, no. 1, pp. 141-145, 1977.

[16] A. Rajasekaran and P. P. Thampi, "Synthesis and analgesic evaluation of some 5-[ $\beta$-(10-phenothiazinyl) ethyl]-1-(acyl)1,2,3,4-tetrazoles," European Journal of Medicinal Chemistry, vol. 39, no. 3, pp. 273-279, 2004.

[17] N. R. Kamdar, D. D. Haveliwala, P. T. Mistry, and S. K. Patel, "Design, synthesis and in vitro evaluation of antitubercular and antimicrobial activity of some novel pyranopyrimidines," European Journal of Medicinal Chemistry, vol. 45, no. 11, pp. 5056-5063, 2010.

[18] D. Hocková, A. Holý, M. Masojídková et al., "Synthesis and antiviral activity of 2,4-diamino-5-cyano-6-[2- (phosphonomethoxy)ethoxy]pyrimidine and related compounds," Bioorganic and Medicinal Chemistry, vol. 12, no. 12, pp. 3197-3202, 2004.

[19] R. Lin, S. G. Johnson, P. J. Connolly et al., "Synthesis and evaluation of 2,7-diamino-thiazolo[4,5- $d$ ] pyrimidine analogues as anti-tumor epidermal growth factor receptor (EGFR) tyrosine kinase inhibitors," Bioorganic and Medicinal Chemistry Letters, vol. 19, no. 8, pp. 2333-2337, 2009.

[20] A. A. Aly, M. Ramadan, A. M. Mohamed, and E. A. Ishak, "Thieno[2, 3-d]pyrimidines in the synthesis of new fused heterocyclic compounds of prospective antitumor and antioxidant agents," Journal of Heterocyclic Chemistry, vol. 49, no. 5, pp. 1009-1018, 2012.

[21] M. T. Chhabria, H. G. Bhatt, H. G. Raval, and P. M. Oza, "Synthesis and biological evaluation of some 5-ethoxycarbonyl6-isopropylamino-4-(substitutedphenyl)aminopyrimidines as potent analgesic and anti-inflammatory agents," Bioorganic and Medicinal Chemistry Letters, vol. 17, no. 4, pp. 1022-1024, 2007.

[22] Y. Kotaiah, N. Harikrishna, K. Nagaraju, and C. V. Rao, "Synthesis and antioxidant activity of 1,3,4-oxadiazole tagged thieno[2, 3-d]pyrimidine derivatives," European Journal of Medicinal Chemistry, vol. 58, pp. 340-345, 2012.

[23] A. M. Marini, F. da Settimo, S. Salerno et al., "Synthesis and in vitro antiproliferative activity of new substituted benzo $\left[3^{\prime}, 2^{\prime}: 5,6\right]$ thiopyrano[ $[4,3-d]$ pyrimidines," Journal of Heterocyclic Chemistry, vol. 45, no. 3, pp. 745-749, 2008.

[24] A. Agarwal, R. Ramesh, A. Ashutosh, N. Goyal, P. M. S. Chauhan, and S. Gupta, "Dihydropyrido[2,3-d]pyrimidines as a new class of antileishmanial agents," Bioorganic and Medicinal Chemistry, vol. 13, no. 24, pp. 6678-6684, 2005.

[25] J. K. Landquist, A. R. Katritzky, and C. W. Rees, Comprehensive Heterocyclic Chemistry, vol. 1, Pergamon Press, Oxford, UK, 1984.

[26] U. Rudolph and H. Möhler, "GABA-based therapeutic approaches: GABAA receptor subtype functions," Current Opinion in Pharmacology, vol. 6, no. 1, pp. 18-23, 2006.

[27] L. Costantino and D. Barlocco, "Privileged structures as leads in medicinal chemistry," Current Medicinal Chemistry, vol. 13, no. 1, pp. 65-85, 2006. 
[28] S. Kanakaraju, B. Prasanna, S. Basavoju, and G. V. P. Chandramouli, "Ionic liquid catalyzed one-pot multi-component synthesis, characterization and antibacterial activity of novel chromeno[2, 3-d]pyrimidine-8-amine derivatives," Journal of Molecular Structure, vol. 1017, pp. 60-64, 2012.

[29] S. Kanakaraju, B. Prasanna, and G. V. P. Chandramouli, "An efficient one-pot three-component synthesis of novel sulfanyl tetrazoles using ionic liquids," Journal of Chemistry, vol. 2013, Article ID 104690, 6 pages, 2013.

[30] S. Abdolmohammadi and S. Balalaie, "Novel and efficient catalysts for the one-pot synthesis of 3,4-dihydropyran$\mathrm{o}[c]$ chromene derivatives in aqueous media," Tetrahedron Letters, vol. 48, no. 18, pp. 3299-3303, 2007.

[31] P. R. Murray, E. J. Baron, M. A. Pfaller et al., Manual of Clinical Microbiology, American Society of Microbiology, Washington, DC, USA, 1995. 

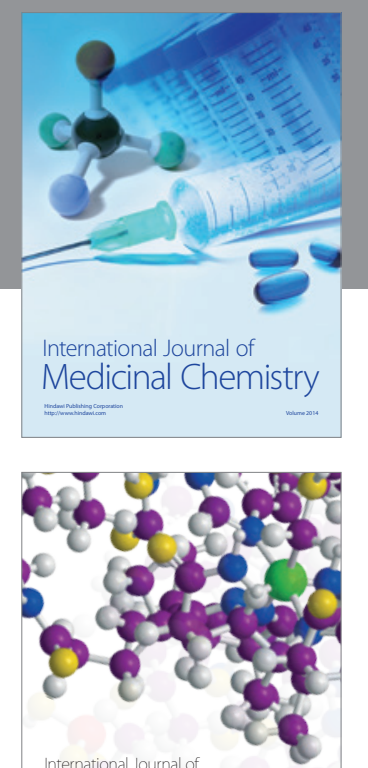

\section{Carbohydrate} Chemistry

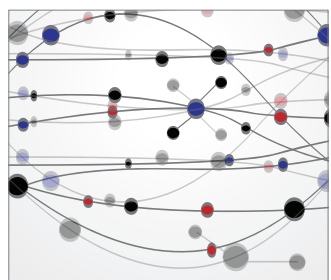

The Scientific World Journal
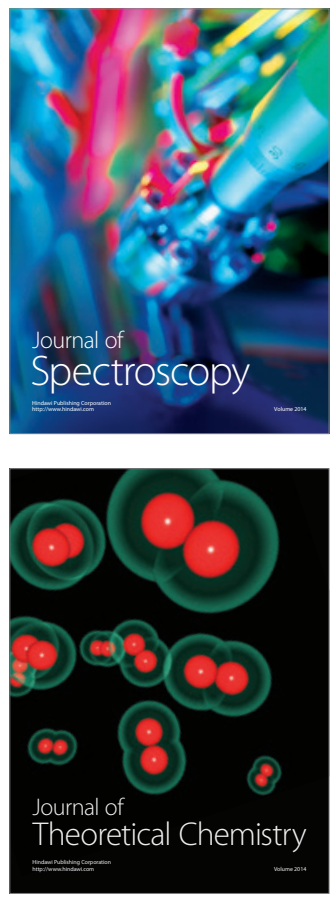
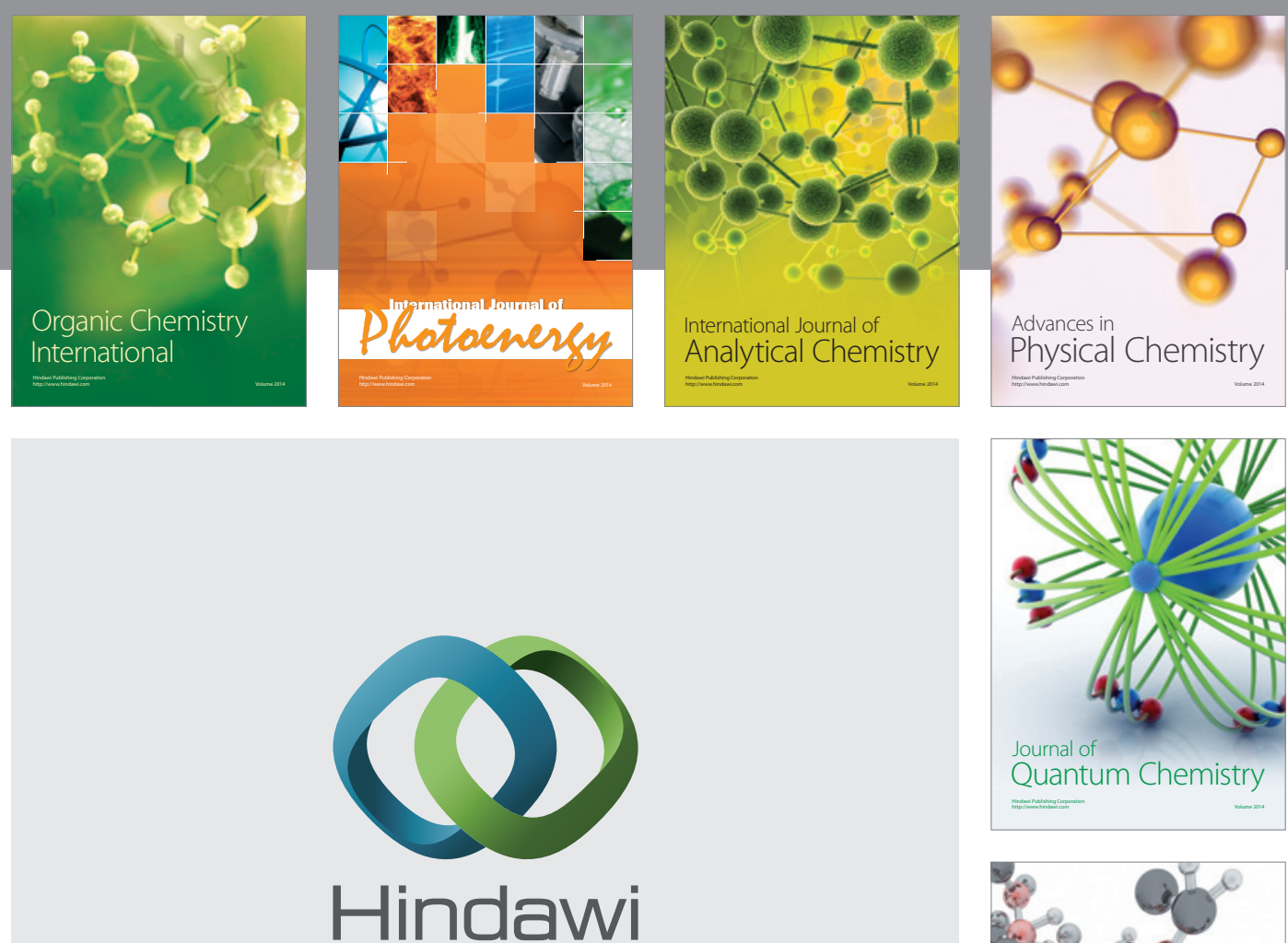

Submit your manuscripts at

http://www.hindawi.com

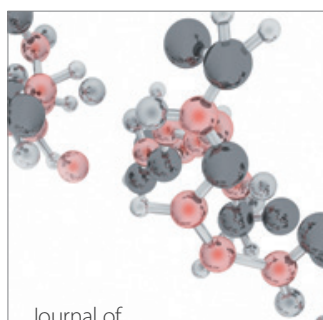

Analytical Methods

in Chemistry

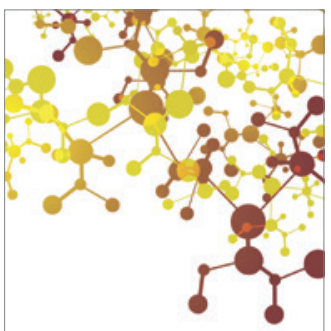

Journal of

Applied Chemistry

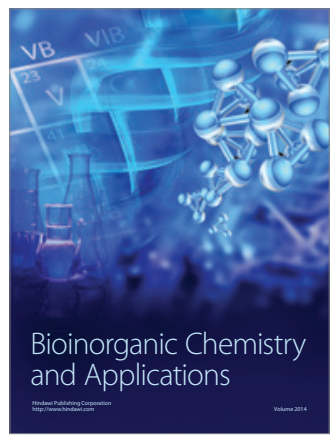

Inorganic Chemistry
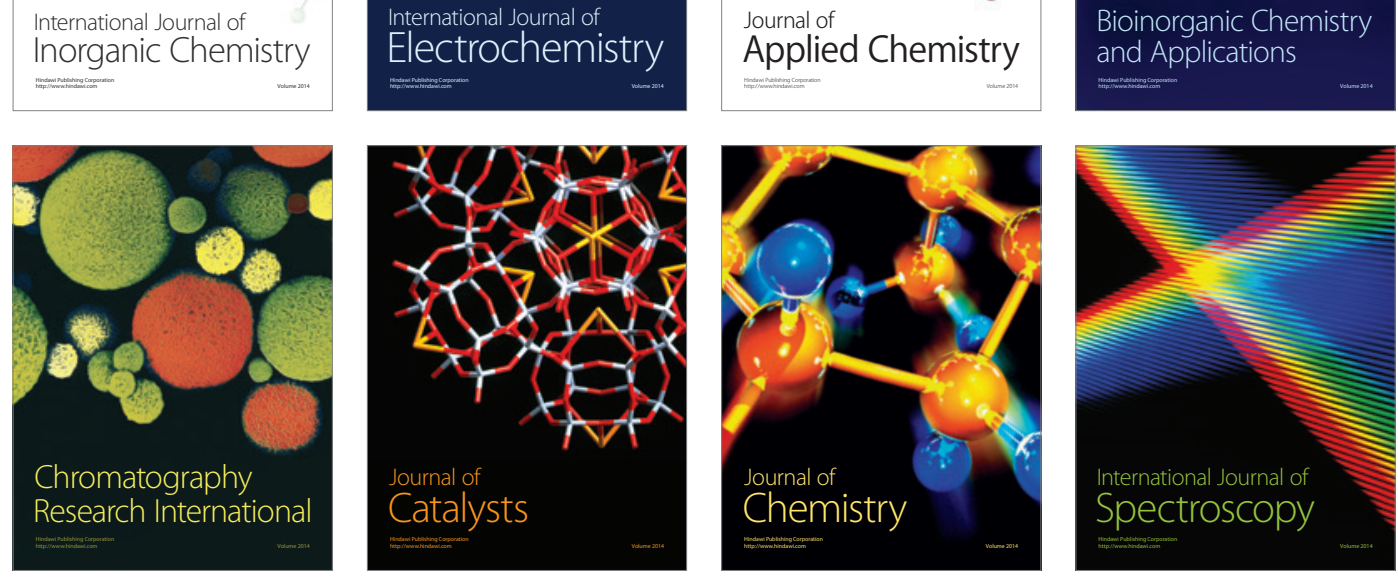